\title{
Joint Affine and Illumination Estimation Using Scale Manipulation Features
}

\author{
Kobi Bentolila ${ }^{1}$ and Joseph M. Francos ${ }^{1}$ \\ Electrical and Computer Engineering Department, \\ Ben-Gurion University, Beer Sheva 84105, Israel
}

\begin{abstract}
We present a novel image transform called Scale Manipulation Features (SMF). The transform calculates affine invariant features of objects in a global manner and avoids using any sort of edge detection. The transform can be used for registration of affine transformed images in the presence of non homogenous illumination changes and for estimation of the illumination changes. The computational load of the method is relatively low since it is linear in the data size. In this paper we introduce the transform and demonstrate its applications for illumination compensation and for object registration in the presence of an affine geometric transformation and varying illumination.
\end{abstract}

Keywords: Geometric distortion, Affine invariance, Illumination invariance, Affine invariant features, image transform.

\section{Introduction}

The general problem of object registration when seen from different points of view and under different illumination conditions is a very complex problem in computer vision. In many cases, the change in point of view is modeled as a geometric transformation like shift, rotation or scale of the image coordinates. Illumination changes, on the other hand can be modeled by a linear combination of basis images.

The idea of representing illumination changes by a linear combination of images was proposed by Shashua [1]. Hallinan [2] represented illumination changes by a linear combination of base images, and used PCA to find an approximation of the basis images. Belhumer and Kreigman [3] showed that the illumination cone of a convex lambertian surface can be constructed from as few as three images. Further work was done by Basri and Jacobs [4] who used spherical harmonics to show that set of images produced by a lambertian surface lies close to a 9 dimensional space.

The most popular methods for image registration today are local methods, such as IBR and EBR [5], SIFT [6], and MSER [7]. These methods identify features of small regions in the image and extract the transformation from the correspondence of the features. Most local methods can handle small non uniform illumination changes but they are sensitive to changes in light source direction. Since the features are local they are sensitive to noise. 
Global methods for affine invariant features include MSA [8], affine invariant moments [9], CW moments [10] and trace transform [11. However, None of these methods can handle illumination changes. Affine and illumination invariance features are presented in [12] however the illumination changes are global and not location dependent.

A global method for registration in the presence of general a multi dimensional geometric homeomorphism was proposed by [13. The method was extended in [14 to affine registration with illumination variation using hyper-spectral data.

In this paper we propose a novel transform called Scale Manipulation Features $(\mathrm{SMF})$. The transform calculates features of isolated objects in a global manner and does not rely on any type of edge detection. The transform can be used for illumination compensation and for registration in the presence of an affine geometric transformation. The computational complexity of the transform is relatively low and grows linearly with the size of the object.

The paper is organized as follows: Section 2 defines the problem of estimating jointly the illumination and affine geometric changes. Section 3 defines the SMF transform, shows its invariance to affine geometric transformations and the linear properties of the transformation. Section 4 describes the illumination estimation scheme. Section [5] defines SMF moments and demonstrates their usage for affine registration. Section 6 describes an experiment of affine registration under varying illumination of real images.

\section{Problem Definition}

In this section we introduce the mathematical model used to describe the problem of object registration in the presence of varying illumination. The mathematical model described here was first defined at [13] and [14].

We begin by defining the geometric deformation. Let $\mathrm{G}$ be the group of the allowed geometric deformations and $S=\left\{f: R^{2} \rightarrow R\right\}, f$ is integrable with bounded support, be the space of the image functions. The action of $G$ on $S$ is defined by $G \times S \rightarrow S$ such that for $\phi \in G, f \in S, \phi$ acts on $f$ by $(\phi, f) \equiv f \circ \phi$ (composition of functions). Loosely speaking, each member of $G$ acts on an image $f$ by changing the coordinates system of $f$. Thus, in the absence of illumination variation, the registration problem is formulated as follows: Given the functions (images) $h$ and $f$, such that $h=f \circ \phi$, find $\phi$.

We will restrict the discussion to affine geometric transformation. We define $G_{a f f}$ to be the group of affine invertible transformation $G=\{\phi(\mathbf{A}, \mathbf{b}) ; \mathbf{A} \in$ $\left.G L(2), \mathbf{b} \in R^{2}\right\}$, where $\phi(\mathbf{x})=\mathbf{A} \mathbf{x}+\mathbf{b}$. The group action of $\phi(\mathbf{A}, \mathbf{b})$ is defined as $(\phi, f)(\mathbf{x})=f(\mathbf{A x}+\mathbf{b})$.

The radiometric changes are modeled as different linear combinations of a number of basis images. Let by $f_{i}, \mathrm{i}=1, \ldots, \mathrm{n}$ be a set of basis functions, then $f=\sum_{i=1}^{n} a_{i} f_{i}$ describes the image of an object viewed at a certain illumination state. 


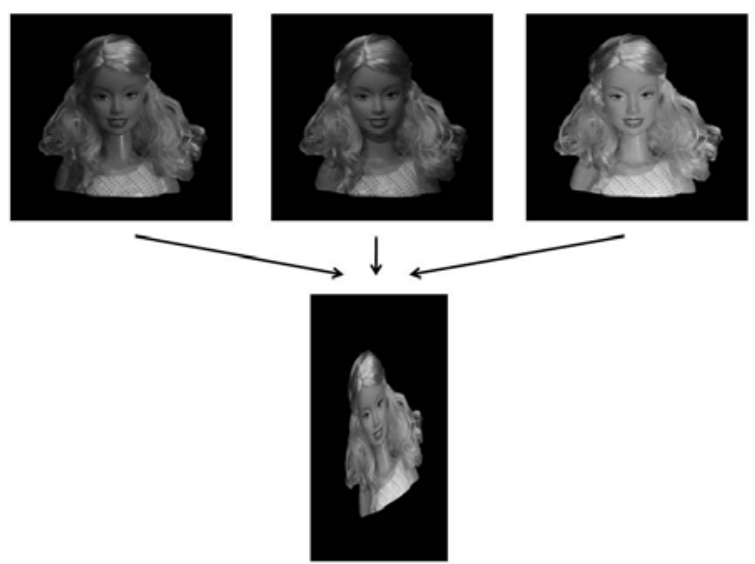

Fig. 1. Different illuminations of an image and an affine transformed linear combination

The problem of joint affine geometric transformation estimation and radiometric change estimation discussed in this paper is therefore defined as

$$
h=\left(\sum_{i=1}^{n} a_{i} f_{i}\right) \circ \phi
$$

Given only the observation $h$ and the illumination basis images $f_{i}, \mathrm{i}=1, \ldots, \mathrm{n}$ we wish to find the coefficients $a_{i}$ and the geometric transformation $\phi$. Figure 1 display an affine transformed mixture of illuminated images.

We use the same notations $\phi, f, f_{i}, h$ throughout this paper to describe the affine transformation, the illuminated image, the basis illumination functions and the observation respectively.

\section{Scale Manipulation Features}

In this section, we introduce an affine invariant image transform called Scale Manipulation Features (SMF) and explain the properties of the transform.

\subsection{The Definition of SMF}

Let

$$
\operatorname{sp}_{f}(\mathbf{x})=\left\{\begin{array}{l}
1 \text { if } f(\mathbf{x})>0 \\
0 \text { else }
\end{array}\right.
$$

be the support function of an image intensity function $f, \mathbf{x} \in R^{2}$. We define the center of mass of the support by

$$
\mu_{f}=\frac{1}{\left\|s p_{f}\right\|_{L_{1}}} \int_{R^{2}} \mathbf{x} s p_{f}(\mathbf{x}) d \mathbf{x}
$$


Let $\alpha$ be a scale factor, $\alpha \in(0,1]$ and let

$$
s p_{f, \alpha}(\mathbf{x})=s p_{f}\left(\frac{1}{\alpha}\left(\mathbf{x}-\mu_{f}\right)+\mu_{f}\right)
$$

be the scale of $s p_{f}$ around the center of mass $\mu_{f}$. Using the above definition we can now define the SMF transform

$$
F(\alpha)=\frac{1}{\left\|s p_{f}\right\|_{L_{1}}} \int_{R^{2}} f(\mathbf{x}) \cdot s p_{f, \alpha}(\mathbf{x}) d \mathbf{x}
$$

The SMF transformation calculates the weight of an image intensity function in regions determined by $\alpha$. Fig 2 shows an illustration of the selected region. Each $\alpha$ value correspond to a region centered at the image support center of mass.
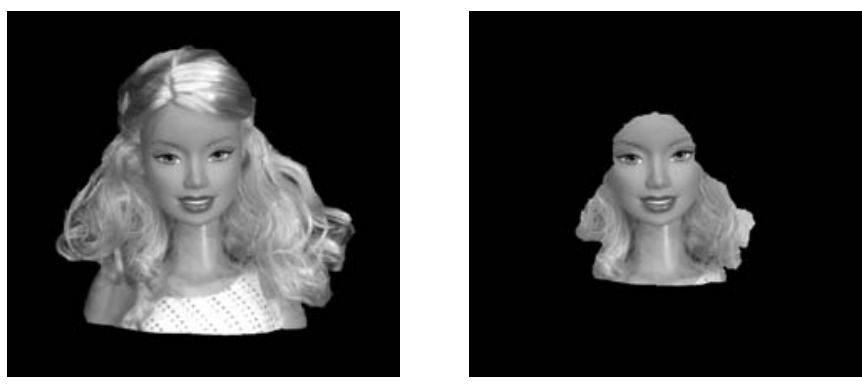

Fig. 2. Original image and scaled integration area, $\alpha=0.6$

\subsection{SMF Invariance to Affine Transformation}

Let $f$ be an image intensity function, $\phi(\mathbf{A}, \mathbf{b})$ an affine transformation and $h=$ $f \circ \phi$ the affine transformed image. Let $F(\alpha)$ and $H(\alpha)$ denote the SMF of $f$ and $h$ respectively.

Theorem 1. $F(\alpha)=H(\alpha)$

Proof. See [15].

Since $F(\alpha)=H(\alpha)$ regardless of the affine transformation, we can use the SMF to extract affine invariant features. Each value of $\alpha$ yields an additional feature.

\subsection{SMF Linear Properties}

Let $f_{i}, \mathrm{i}=1, \ldots, \mathrm{n}$ be some image intensity functions representing an object in the same pose under different illuminations, and let $F_{i}(\alpha)$ be their corresponding SMF transforms. Let $a_{i}, \mathrm{i}=1, . ., \mathrm{n}$ be a set of non negative coefficients, and let $f=\sum_{i=1}^{n} a_{i} f_{i}$ be a linear combination of the images, representing the object under some arbitrary illumination condition. As above, let $F(\alpha)$ denote the SMF transform of $f$. 
Theorem 2. $F(\alpha)=\sum_{i=1}^{n} a_{i} F_{i}(\alpha)$

Proof.

$F(\alpha)=\frac{1}{\left\|s p_{f}\right\|_{L_{1}}} \int_{R^{2}} \sum_{i=1}^{n} a_{i} f_{i}(\mathbf{x}) \cdot s p_{f, \alpha}(\mathbf{x}) d \mathbf{x}=\sum_{i=1}^{n} a_{i} \frac{1}{\left\|s p_{f}\right\|_{L_{1}}} \int_{R^{2}} f_{i}(\mathbf{x}) \cdot s p_{f, \alpha}(\mathbf{x}) d \mathbf{x}$

Since $f_{i}$ represents the object in the same pose, under a different illumination, we have that for each $\mathrm{i}, \mathrm{j} \in\{1, \ldots, \mathrm{n}\}, s p_{f_{i}}=s p_{f_{j}}=s p_{f}$. Therefore

$$
F(\alpha)=\sum_{i=1}^{n} a_{i} \frac{1}{\left\|s p_{f_{i}}\right\| L_{1}} \int_{R^{2}} f_{i}(\mathbf{x}) \cdot s p_{f_{i}, \alpha}(\mathbf{x}) d \mathbf{x}=\sum_{i=1}^{n} a_{i} F_{i}(\alpha)
$$

\section{SMF for Estimating Illumination in the Presence of Affine Transformation}

By theorems 1 and 2

$$
H(\alpha)=\sum_{i=1}^{n} a_{i} F_{i}(\alpha)
$$

Let $\alpha_{1}, \ldots, \alpha_{m}$ be a set of $m$ different coefficients. Evaluating the SMF in (6) for these coefficients results in the following set of linear equations in $a_{1}, \ldots a_{m}$

$$
\left(\begin{array}{ccc}
F_{1}\left(\alpha_{1}\right) & \cdots & F_{n}\left(\alpha_{1}\right) \\
\vdots & \ddots & \vdots \\
F_{1}\left(\alpha_{m}\right) & \cdots & F_{n}\left(\alpha_{m}\right)
\end{array}\right) \cdot\left(\begin{array}{c}
a_{1} \\
\vdots \\
a_{n}
\end{array}\right)=\left(\begin{array}{c}
H\left(\alpha_{1}\right) \\
\vdots \\
H\left(\alpha_{m}\right)
\end{array}\right)
$$

In the absence of noise, using $m=n$ coefficients provides a linear system with $\mathrm{n}$ equations and $\mathrm{n}$ unknowns that can be solved by matrix inversion. In the presence of noise, using $m>n$ equations leads to a linear regression problem. An estimation of $a_{1}, \ldots, a_{n}$ is then obtained by a least square method.

Once $a_{1}, \ldots, a_{n}$ are known, the illumination changes can be compensated for and the problem is reduced to a strictly geometric form of $h=f \circ \phi$ where $f$ is known and is given by $f=\sum_{i=1}^{n} a_{i} f_{i}$.

\section{Affine Transformation Estimation Using First and Second Order SMF Moments}

We define the first order moment SMF of $f, \mathbf{F}^{1}(\alpha), \mathbf{F}^{1} \in R^{2}$ as

$$
\mathbf{F}^{1}(\alpha)=\frac{1}{\left\|s p_{f}\right\|_{L_{1}}} \int_{R^{2}}\left(\mathbf{x}-\mu_{f}\right) \cdot f(\mathbf{x}) \cdot s p_{f, \alpha}(\mathbf{x}) d \mathbf{x}
$$

and the second order moment SMF of $f, \mathbf{F}^{2}(\alpha), \mathbf{F}^{2} \in R^{2 \times 2}$ as

$$
\mathbf{F}^{2}(\alpha)=\frac{1}{\left\|s p_{f}\right\|_{L_{1}}} \int_{R^{2}}\left(\mathbf{x}-\mu_{f}\right) \cdot\left(\mathbf{x}-\mu_{f}\right)^{T} \cdot f(\mathbf{x}) \cdot s p_{f, \alpha}(\mathbf{x}) d \mathbf{x}
$$

The first and second order moments SMF are the first and second order moments of the image function at regions determined by $\alpha$. 
Theorem 3. $\boldsymbol{F}^{1}(\alpha)=\boldsymbol{A} \cdot \boldsymbol{H}^{1}(\alpha)$

Theorem 4. $\boldsymbol{F}^{2}(\alpha)=\boldsymbol{A} \cdot \boldsymbol{H}^{2}(\alpha) \cdot \boldsymbol{A}^{T}$

Proof. See [15.

The second order moment SMF does not lead directly to linear constraints, but linear constraints can be derived from it: Using theorem 4 we have

$$
\mathbf{F}^{2}(\alpha) \cdot \mathbf{A}^{-T}=\mathbf{A} \cdot \mathbf{H}^{2}(\alpha)
$$

Using the notation $\mathbf{A}=\left(\begin{array}{ll}a_{11} & a_{12} \\ a_{21} & a_{22}\end{array}\right)$, the matrix $A^{-T}$ can be written as

$$
\mathbf{A}^{-T}=\frac{1}{|\operatorname{det}(A)|}\left(\begin{array}{cc}
a_{22} & -a_{21} \\
-a_{12} & a_{11}
\end{array}\right)=\frac{\left\|s p_{h}\right\|_{L_{1}}}{\left\|s p_{f}\right\|_{L_{1}}}\left(\begin{array}{cc}
a_{22} & -a_{21} \\
-a_{12} & a_{11}
\end{array}\right)
$$

Thus first and second order moments SMF yield six linear constraints on the values of $\mathbf{A}$ for each value of $\alpha$. By using linear methods such as least squares we can extract the parameters of the matrix $\mathbf{A}$. Once the parameters of $\mathbf{A}$ are known, the shift term $\mathbf{b}$ can be easily calculated.

\section{Experiment - Joint Estimation of Illumination and Affine Geometric Deformation}

In this experiment we demonstrate SMF ability for registration of images in presence of location dependent illumination changes.

\subsection{Experiment Settings}

We use an array of 14 light sources to illuminate a glossy toy airplane. PCA is performed on 100 gray-scale images of the airplane. The images are taken at the same pose, with a random illumination power of each light source distributed uniformly in the range of $10 \%$ to $90 \%$ of the maximal power. 10 basis images, $f_{1}, . ., f_{10}$ are extracted from the PCA.
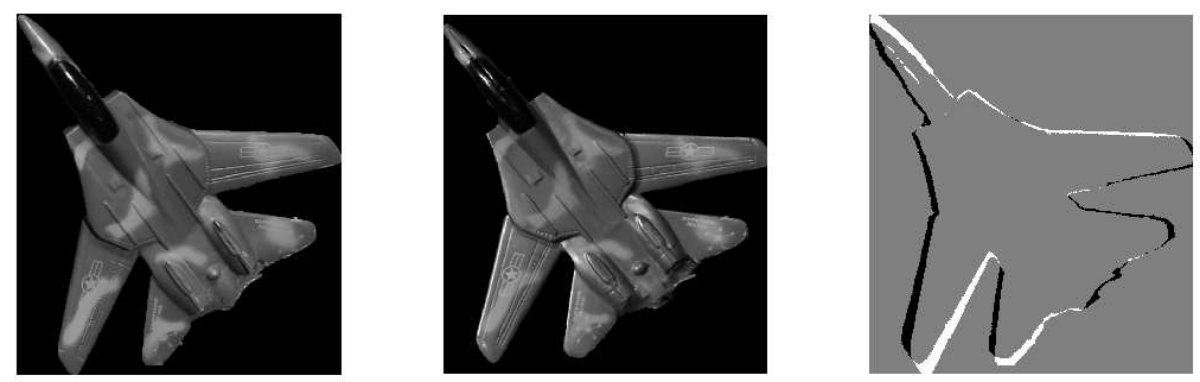

Fig. 3. Registration results - rotation -40 , pan -30 

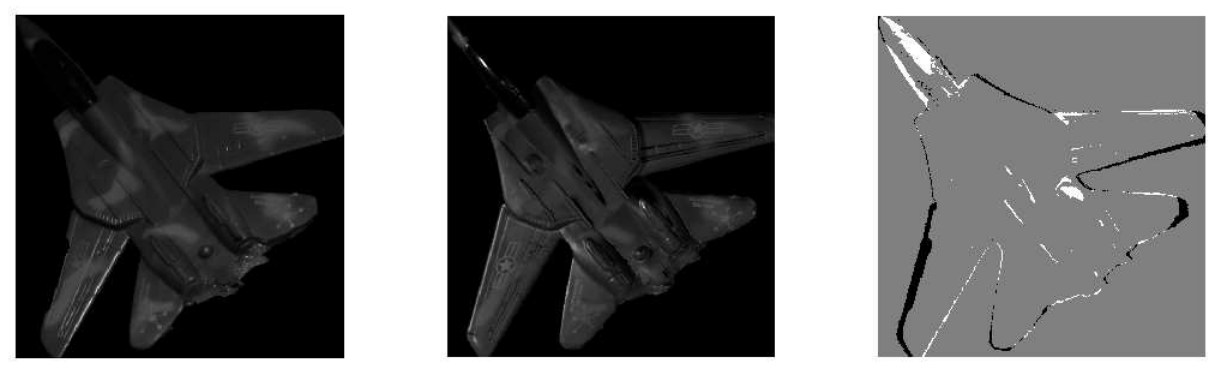

Fig. 4. Registration results - rotation -40 , pan 30
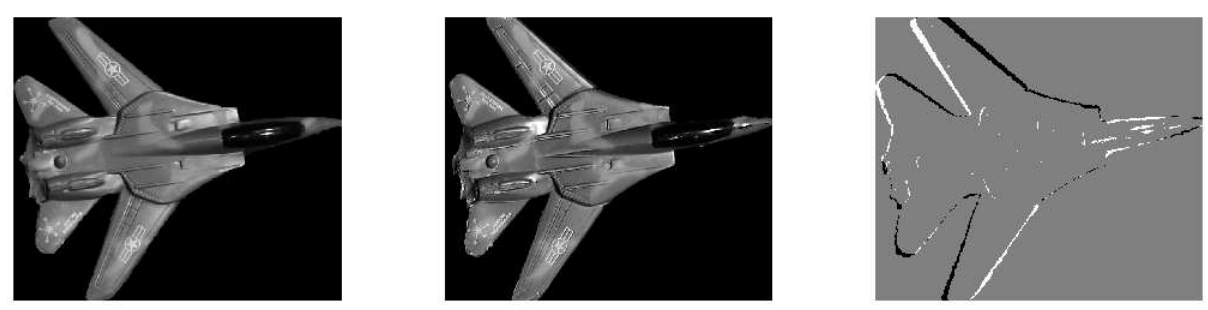

Fig. 5. Registration results - rotation 80 , pan 0

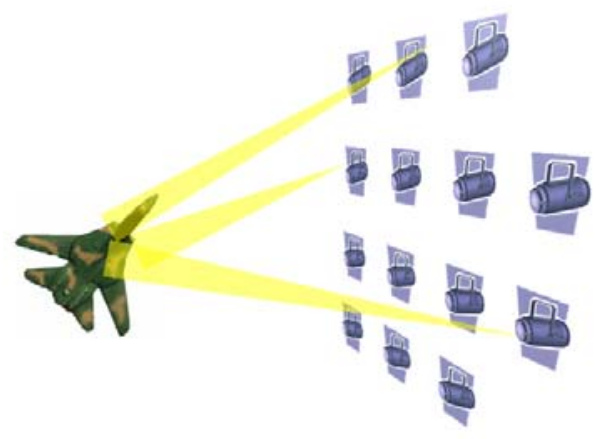

Fig. 6. Illustration of the illuminated plane

We connect the plane to a two axis motorized stage for rotation and pan. The observations $h$ are images of the plane taken at various poses, in each image the illumination power of each of light source is chosen randomly as above. The registration problem is described as $h=\left(\sum_{i=1}^{n} a_{i} f_{i}\right) \circ \phi$.

\subsection{The Registration Process}

For each image, we first estimate the illumination coefficients, $a_{i}$, using the method described in section 4 and reduce the problem to the form $h=f \circ \phi$, where $\widehat{f}=\sum_{i=1}^{10} a_{i} f_{i}$. After the illumination changes are compensated for, an 
estimation of the affine transformation, $\widehat{\phi}$, is performed using the constraints described is section 5 .

The registration results of various illumination and pose conditions are illustrated in figures 34 and 5. The leftmost images show the observations, $h$. The middle images show $\widehat{h}=\widehat{f} \circ \widehat{\phi}$ - the estimated affine transform applied to the estimated illuminated template image. The rightmost images show the difference image of the support between the observation $h$ and $\widehat{h}$.

\section{Conclusion}

We have introduced a novel image transformation, SMF, and demonstrated its properties and usage. We showed that the transformation can be used for registration in the presence of harsh illumination changes. The method is simple and requires only integrations of the image and linear equations solving, therefore it is computationally inexpensive.

\section{References}

1. Shashua, A.: Geometry and Photometry in 30 Visual Recognition. PhD thesis, MIT Dept of Brain and Cognitive Science (August 1992)

2. Hallinan, P.W.: A low-dimensional representation of human faces for arbitrary lighting conditions. In: Proceedings of the IEEE Conference on Computer Vision and Pattern Recognition, pp. 995-999 (1994)

3. Belhumeur, P., Kriegman, D.: What Is the Set of Images of an Object under All Possible Lighting Conditions. Intl J. Computer Vision 28, 245-260 (1998)

4. Basri, R., Jacobs, D.W.: Lambertian Reflectance and Linear Subspaces. IEEE Transactions on Pattern Analysis and Machine Intelligence 25(2), 218-233 (2003)

5. Tuytelaars, T., Van Gool, L.: Matching Widely Separated Views Based on Affine Invariant Regions. Intl J. Computer Vision 1(59), 61-85 (2004)

6. Lowe, D.G.: Distinctive image features from scale-invariant keypoints. International Journal of Computer Vision 60(2), 91-110 (2004)

7. Matas, J., Chum, O., Urban, M., Pajdla, T.: Robust wide baseline stereo from maximally stable extremal regions. In: Proc. of British Machine Vision Conference, pp. 384-396 (2002)

8. Rahtu, E., Salo, M., Heikkila, J.: Affine invariant pattern recognition using multiscale autoconvolution. IEEE Transactions on Pattern Analysis and Machine Intelligence 27(6), 908-918 (2005)

9. Flusser, J., Suk, T.: Pattern recognition by affine moment invariants. Pattern Recognition 26(1), 167-174 (1993)

10. Yang, Z., Cohen, F.S.: Cross-weighted moments and affine invariants for image registration and matching. IEEE Trans. Pattern Analysis and Machine Intelligence 21(8), 804-814 (1999)

11. Petrou, M., Kadyrov, A.: Affine invariant features from the trace transform. IEEE Trans. Pattern Analysis and Machine Intelligence 26(1), 30-44 (2004) 
12. Mindru, F., Tuytelaars, T., van Gool, L., Moons, T.: Moment Invariants for Recognition under Changing Viewpoint and Illumination. Computer Vision and Image Understanding 94, 3-27 (2004)

13. Francos, J.M., Hagege, R., Friedlander, B.: Estimation of Multi-Dimensional Homeomorphisms for Object Recognition in Noisy Environments. In: Thirty Seventh Asilomar Conference on Signals, Systems, and Computers (2003)

14. Frenkel, R., Francos, J.: Registration of geometric deformations in the presence of varying illumination. In: Proc. IEEE International Conference on Image Processing 2007, vol. 3, pp. 489-492 (2007)

15. Bentolila, K., Francos, J.: Affine and Illumination Estimation Using Scale Manipulation Transform (to appear) 